\title{
Extraction of Aminoglycoside Antibiotics with Reverse Micelles
}

\author{
Ziyi Hu \& Erdogan Gulari* \\ Department of Chemical Engineering, The University of Michigan, Ann Arbor, Michigan, USA \\ (Received 11 May 1995; revised version received 1 August 1995; accepted 24 August 1995)
}

\begin{abstract}
The reverse micelle system of sodium di-2-ethylhexyl phosphate was used to extract aminoglycoside antibiotics, neomycin and gentamicin. The aminoglycosides can be efficiently extracted into a reverse micelle solution, and the antibiotics extracted into the micelle phase can readily be recovered back to a divalent cation aqueous solution, such as $\mathrm{Ca}^{2+}$. The transfer efficiency, $\% E$, is heavily dependent on $\mathrm{pH}$ and salt concentration in the aqueous feed solution. $\% E$ decreases drastically with $\mathrm{pH}$ in the $\mathrm{pH}$ range $8 \cdot 5-11$, and declines with increasing $\left(\mathrm{NH}_{4}\right)_{2} \mathrm{SO}_{4}$ concentration. A simple transfer mechanism was proposed which suggests that the antibiotic molecules were extracted into the inner water cores of reverse micelles through attractive electrostatic interaction during forward transfer. In backward transfer, the antibiotics loaded in the micelle phase are released back to an aqueous phase through breaking up of the reverse micelles by using divalent cation solutions. The model is supported by the results of dynamic light scattering and infra-red spectroscopy study.
\end{abstract}

Key words: antibiotics, extraction, reverse micelles.

\section{INTRODUCTION}

The use of reverse micelle systems for bioseparation has received considerable attention in recent years. The systems which have some attractive advantages for whole broth processing, consist of an aqueous phase of bioproducts and an organic solution containing reverse micelles. The 'water pool' of the reverse micelle inner core can serve as a carrier for the bioproducts. While the aqueous phase is contacted with the organic reverse micelles phase, the bio-molecules can be transferred from the aqueous phase to the polar core of reverse micelles, mainly because of the attractive electrostatic interaction between the inner micelle charge wall and the bio-molecules. By manipulating the operation conditions, the products extracted into the organic phase can be recovered back to an aqueous phase. In theory, the reverse micelle system is easy to scale up, feasible for continuous processing and simple in operation. Numerous studies have been carried out in $\operatorname{protein}^{1-4}$ and amino acid $^{5-8}$ extraction with this system. Aminoglycosides are characterized by carbohydrate moieties bound to complex cyclitol structures. Conventional

* To whom correspondence should be addressed. organic solvent extraction is ineffective for these molecules because of their hydrophilic nature, and ion exchange is the primary method used for recovery of these products in industrial operation. ${ }^{9}$ Since aminoglycosides possess positive charges at neutral $\mathrm{pH}$, these molecules, theoretically, could be extracted from a fermentation broth into an anionic surfactant reverse micelle phase. In the present work, we used the reverse micelle system of sodium di-2-ethylhexyl phosphate (NaDEHP, an anionic surfactant) to extract two commercially important aminoglycoside antibiotics, neomycin and gentamicin.

\section{EXPERIMENTAL}

\section{Materials}

Neomycin and gentamicin (purity over 90\% and 99\%, respectively) were obtained from Fluka. Di-2-ethylhexyl phosphate (HDEHP) was obtained from Aldrich and was over $97 \%$ pure. Tributyl phosphate (TBP), which was used as a cosurfactant, was obtained from Fluka and was over $99 \%$ pure. The organic solvent used, isooctane, was spectroscopic grade and deionized water was used to prepare all aqueous solutions. 


\section{Reverse micelle phase preparation}

The reverse micellar solution was prepared by mixing equal volumes of $\mathrm{HDEHP} /$ isooctane solution with an equal molar concentration of $\mathrm{NaOH}$ aqueous solution to neutralize the phosphoric acid. TBP was added as a cosurfactant to the organic phase with a TBP/HDEHP ratio of 3 , and $0.2 \mathrm{M} \mathrm{NaCl}$ was also added to the aqueous phase. The $\mathrm{pH}$ of the aqueous phase was adjusted with $0.1 \mathrm{M} \mathrm{NaOH}$ and $0.1 \mathrm{M} \mathrm{HCl}$ aqueous solutions to about 8 . After phase separation, NaDEHP reverse micelle phase was formed in the upper phase.

\section{Forward extraction}

Forward extraction experiments were performed by contacting $2 \mathrm{~cm}^{3}$ of $200 \mathrm{~mm}$ NaDEHP reverse micelle solution with $2 \mathrm{~cm}^{3}$ of an antibiotic solution of $20 \mathrm{~g} \mathrm{dm}^{-3}$ neomycin or $30 \mathrm{~g} \mathrm{dm}^{-3}$ gentamicin, in a Teflon-capped glass test tube at room temperature $\left(24 \pm 1^{\circ} \mathrm{C}\right)$. The tube was shaken vigorously for $2 \mathrm{~min}$, then centrifuged ( $2000 \mathrm{rpm}, 2 \mathrm{~min}$ ) for phase separation. After centrifugation, both phases were transparent with a distinct phase boundary. The antibiotic concentration in the aqueous phase was measured by UV-visible spectroscopy using the $310 \mathrm{~nm}$ band. Since no precipitate was found at the organic-aqueous interface after forward extraction, the antibiotic concentration in the organic phase was determined by measuring the difference of antibiotic concentrations in the aqueous phase before and after forward transfer. Transfer percentage, $\% E$, is used to represent the transfer efficiency which is defined as the ratio of the antibiotics extracted into the organic phase to the total amount of the antibiotic.

\section{Backward extraction}

Preceding backward transfer, a forward transfer was carried out by mixing $3 \mathrm{~cm}^{3}$ of an aqueous solution of $10 \mathrm{~g} \mathrm{dm}^{-3}$ neomycin and $0.2 \mathrm{M} \mathrm{NaCl}$ with $3 \mathrm{~cm}^{3}$ of micelle phase of $0.1 \mathrm{M} \mathrm{NaDEHP} /$ isooctane. The backward extraction was then performed by mixing equal volumes of $0.1 \mathrm{M} \mathrm{CaCl}$ aqueous solutions with the antibiotic-loaded micelle solution, shaking vigorously for $5 \mathrm{~min}$ and then centrifuging $2000 \mathrm{rpm}, 2 \mathrm{~min}$ ) for phase separation. The basis for this process is to convert $\mathrm{NaDEHP}$ to a divalent metal salt of $\mathrm{M}(\mathrm{DEHP})_{2}$ which is much less surface active than the sodium salt and very soluble in isooctance. As a result, the reverse micelles are broken up and the antibiotic loaded into the micelle phase is then released back to an aqueous phase.

\section{Analytical methods}

Ultraviolet-visible spectroscopic measurements were carried out with an HP8451A diode array spectrophotometer. Dynamic light scattering was used to measure the size of the reverse micelles before and after the forward/backward extraction cycle. The water content in the organic phase, and the concentrations of the surfactant and TBP, before and after forward/ backward transfer, were determined by infra-red spectroscopy.

\section{RESULTS AND DISCUSSION}

\section{Forward transfer}

Under adequate conditions, over $80 \%$ of the antibiotics were transferred to the $\mathrm{NaDEHP}$ reverse micelle phase after a single stage of forward extraction. This evidently shows that aminoglycoside molecules can be extracted into a reverse micelle phase.

The transfer efficiency, $\% E$, is strongly dependent on $\mathrm{pH}$, and the relationship between $\% E$ and $\mathrm{pH}$ is illustrated in Fig. 1. In the study of pH effects, the aqueous antibiotic solutions were prepared by dissolving the antibiotics into $0.05 \mathrm{M}$ Tris- $\mathrm{HCl}$ buffer solutions with $0.3 \mathrm{M} \mathrm{NaCl}$ solutions.

In the $\mathrm{pH}$ range of $8 \cdot 5-11$, the transfer efficiencies sharply decrease with the $\mathrm{pH}$. The decline of $\% E$ with $\mathrm{pH}$ indicates that forward transfer is a result of the attractive electrostatic interaction between the antibiotic molecules and the charged surfactant heads. As the $\mathrm{pH}$ increases, the positive charge of aminoglycoside molecules decreases, and the magnitude of attractive interaction diminishes. Therefore, $\% E$ declines.

The presence of salt in the aqueous solution also affects the transfer of aminoglycosides. The influence of salt $\left(\mathrm{NH}_{4}\right)_{2} \mathrm{SO}_{4}$ on $\% E$ is illustrated in Fig. 2, which shows that $\% E$ decreases with $\left(\mathrm{NH}_{4}\right)_{2} \mathrm{SO}_{4}$ concentration. At $0.2 \mathrm{M}\left(\mathrm{NH}_{4}\right)_{2} \mathrm{SO}_{4}$, the transfer efficiencies of neomycin and gentamicin are $75 \%$ and $82 \%$, respectively. The values of $\% E$ reduce to $13 \%$ and $10 \%$ as the salt concentration increases to $2 \mathrm{M}$.

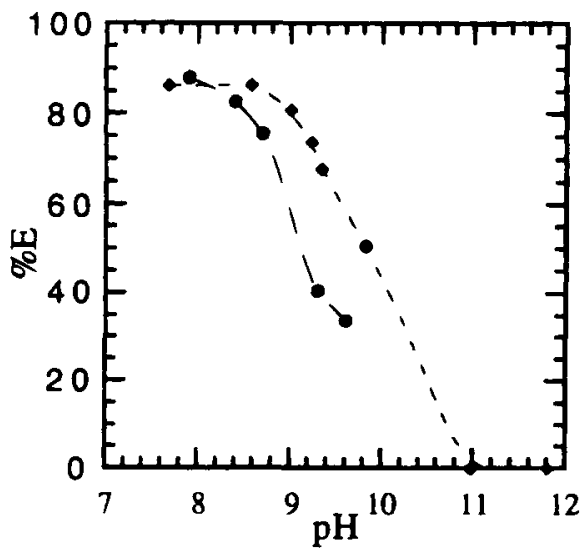

Fig. 1. The transfer efficiency as a function of $\mathrm{pH}$. Neomycin; : gentamicin. 


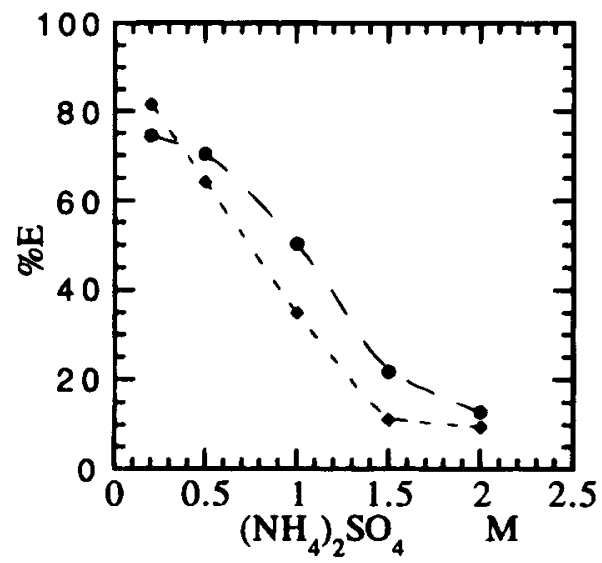

Fig. 2. The variation in transfer efficiencies with $\left(\mathrm{NH}_{4}\right)_{2} \mathrm{SO}_{4}$ : Neomycin; : gentamicin.

With increasing $\left(\mathrm{NH}_{4}\right)_{2} \mathrm{SO}_{4}$ concentration, the attractive electrostatic interaction between surfactant and aminoglycosides attenuates probably because of the Debye screening effect, and this causes the decline of the transfer efficiency.

\section{Backward extraction}

Figure 3 shows the UV spectra of neomycin aqueous solutions before extraction, and the one after the backward transfer. The overall recovery rate is $67.6 \%$. In this case, the transfer efficiency of the forward transfer was $67.7 \%$; thus, nearly $100 \%$ of the neomycin molecules loaded in the micelle phase are recovered back to the aqueous phase.

\section{Mechanisms of forward and backward transfer}

So far, our discussion has implied that the aminoglycosides were extracted into the inner water cores of the reverse micelles during the forward transfer, and

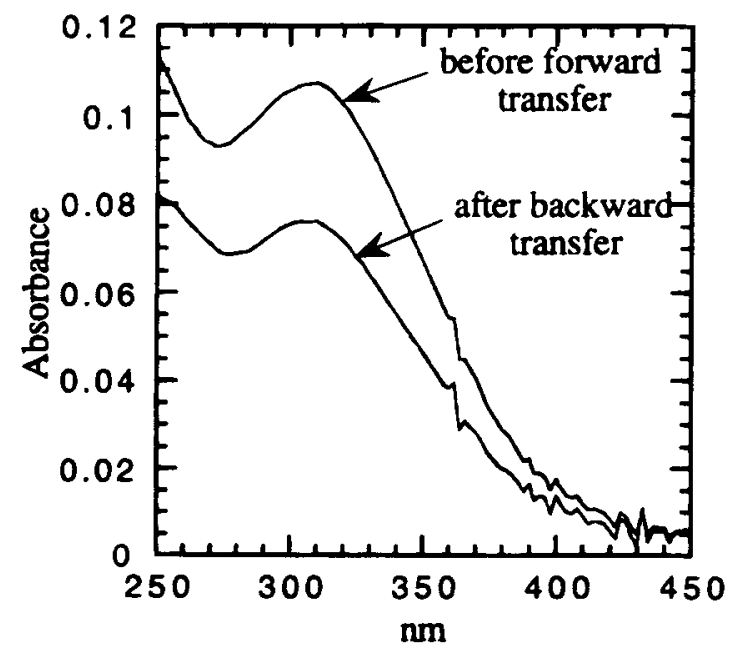

Fig. 3. UV spectra of the neomycin aqueous solutions with initial neomycin concentration of $10 \mathrm{~g} \mathrm{dm}^{-3}$. recovered to an aqueous phase by demolishing the micelle system. Since NaDEHP is a cation exchanger, it is also possible that the aminoglycosides were extracted into the organic phase through forming a hydrophobic aminoglycoside-carrier complex, similar to the reactive solvent extraction of penicillin $\mathrm{G}^{10-12}$ and cephalosporin C. ${ }^{13}$

Dynamic light scattering and infra-red study were carried out to investigate the mechanisms of forward and backward transfer. Dynamic light scattering measurements results show that the diameter of the reverse micelle is $5.5 \mathrm{~nm}$ before forward extraction. After forward transfer, the micelle size decreases to $4.4 \mathrm{~nm}$ in diameter. After backward extraction, no micelles are detected in the upper phase.

The infra-red spectroscopy was used to determine the water contents in the upper phase at different extraction stages. Figure 4 shows the infra-red spectra of the upper phases before and after forward extraction, and after backward transfer. The absorbency band near $3400 \mathrm{~cm}^{-1}$ is from the water uptake to the upper phase. The $1020 \mathrm{~cm}^{-1}$ peak is the $\mathrm{P}-\mathrm{O}-\mathrm{R}$ vibration band which comes from the NaDEHP and TBP molecules. Before forward transfer, a considerable amount of water uptake into the upper phase occurs, thus forming the water cores of the reverse micelles. After forward transfer, the water content decreases slightly, which indicates that the reverse micelles still exist in the upper phase. After backward transfer, the $3400 \mathrm{~cm}^{-1}$ band almost disappears. This indicates that the reverse micelles in the upper phase are destroyed after the backward transfer, and the water is released back to the lower phase. The intensity of the $1020 \mathrm{~cm}^{-1}$ peak essentially remains the same during the forward and backward transfer, which indicates that the losses of the surfactant NaDEHP and modifier TBP are low during the transfer processes.

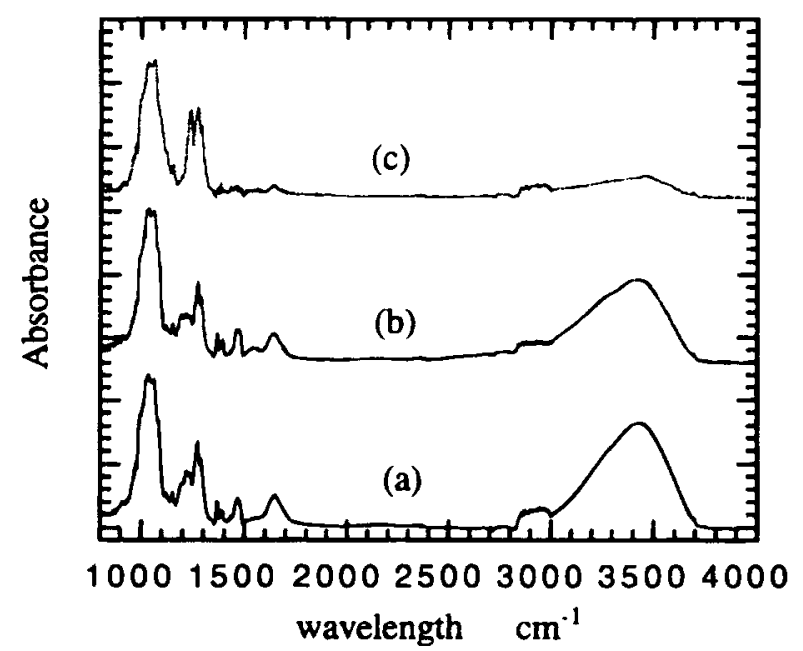

Fig. 4. Infra-red spectra of the organic solutions in the extraction of neomycin. (a) Before transfer; (b) after forward transfer; (c) after backward transfer. 
These results indicate that the aminoglycoside molecules appear to be extracted into the water pool of the reverse micelle cores after forward transfer. The extraction is a result of the attractive electrostatic interaction between the inner micelle wall and the aminoglycoside molecules. During the backward transfer, the reverse micelles are demolished because of the formation of $\mathrm{Ca}(\mathrm{DEHP})_{2}$ which is much less surface active and very soluble in the organic phase. Consequently, the aminoglycoside molecules are recovered back to the aqueous phase.

\section{CONCLUSION}

The aminoglycoside antibiotics neomycin and gentamicin can be efficiently extracted into a reverse micelle solution through the attractive electrostatic interaction between the antibiotic molecules and the charge layer of the inner micelle core. The antibiotics extracted into the micelle phase can readily be recovered back to an aqueous solution. The $\mathrm{pH}$ and salt concentration in the aqueous solution have a strong influence on the forward transfer efficiency. The transfer efficiency, $\% E$, drastically decreases with $\mathrm{pH}$ in the $\mathrm{pH}$ range $8 \cdot 5-11$, and declines with increasing $\left(\mathrm{NH}_{4}\right)_{2} \mathrm{SO}_{4}$ concentration. This study exhibits a promising method in the purification of aminoglycoside antibiotics.

\section{REFERENCES}

1. Luisi, P. L., Bonner, F. J., Pellegrin, A., Wiget, P. \& Wolf, R., Micellar solubilization of proteins in aprotic solvents and their spectroscopic characterization. Helv. Chim. Acta, 62 (1979) 740-53.

2. Goklen, K. E. \& Hatton, T. A., Liquid-liquid extraction of low molecular-weight proteins by selective solubilization in reversed micelles. Sep. Sci. Technol., 22 (1987) 831-41.

3. Dekker, M., Hilhorst, R. \& Laane, C., Isolating enzymes by reversed micelles. Anal. Biochem., 178 (1989) 217-26.

4. Lesser, M. E. \& Luisi, P. L., Application of reverse micelles for the extraction of amino acids and proteins. Chimia, 44 (1990) 270-82.

5. Furusahi, S. \& Kishi, K., Extraction of amino acids by reversed micelles. J. Chem. Eng. Japan, 23 (1990) 91-3.

6. Leodidis, E. B. \& Hatton, T. A., Amino acids in AOT reversed micelles. 1. Determination of interfacial partition coefficients using the phase-transfer method. J. Phys. Chem., 94 (1990) 6400-11.

7. Adachi, M., Harada, M., Shioi, A. \& Sato, Y., Extraction of amino acids to microemulsion. J. Phys. Chem., 95 (1991) 7925-31.

8. Schugerl, K., Solvent Extraction in Biotechnology. Recovery of Primary and Secondary Metabolites. SpringerVerlag, Berlin, Heidelbeg, 1994.

9. Beelter, P. A., Ion exchange recovery of antibiotics. In Comprehensive Biotechnology, eds M. M. Young, C. L. Cooney \& A. E. Humphrey, Pergamon, Oxford, 1985, Vol. 2, pp. 473-80.

10. Reschke, M. \& Schugerl, K., Reactive extraction of penicillin. II: Distribution coefficients and degree of extraction. Chem/Biochem. Eng. J., 28 (1984) B11-B20.

11. Likidis, Z., Schlichting, E., Bishoff, L. \& Schugerl, K., Reactive extraction of penicillin $\mathrm{G}$ from mycel-containing broth in a countercurrent extraction decanter. Biotechnol. Bioeng., 33 (1989) 1385-92.

12. Harris, T. A. J., Khan, S., Reuben, B. G., Shokoya, T. \& Verrall, M., Reactive solvent extraction of beta-lactam antibiotics. In Separation for Biotechnology, ed. D. L. Pyle. Elsevier Applied Science, London, 1990, pp. 172-80.

13. Hano, T., Matsumoto, M., Ohtake, T. \& Hori, F. J., Reactive extraction of cephalosporin C. J. Chem. Eng. Japan, 25 (1992) 293-6. 